\title{
Growth studies of thin films with total scattering in real time
}

\author{
Martin Roelsgaard ${ }^{a}$, Ann-Christin Dippel $^{b}$, Bo Brummerstedt Iversen ${ }^{a}$ \\ ${ }^{a}$ Dept. of Chemistry \& iNANO, Aarhus University, Langelandsgade 140, 8000 Aarhus C, Denmark ${ }^{b}$ PETRA III, Deutsches \\ Elektronen-Synchrotron (DESY), 22607 Hamburg, Germany
}

\section{m.roelsgaard@chem.au.dk}

Thin films of thickness $100 \mathrm{~nm}$ or less are typically deposited on a much thicker substrate, making it difficult to obtain the required high-quality total scattering data for analysis in real-space with pair distribution functions (PDF) [1]. In recent years, total scattering in reflection geometry at grazing incidence (GI), that is, below the critical angle of total external reflection, has been used with success to increase the surface sensitivity and scattering intensity [2,3]. GI-PDF gives high-quality PDFs from films as thin as $3 \mathrm{~nm}$ with a $20 \mathrm{x}$ $2.5 \mu \mathrm{m}^{2}$ focused X-ray beam with $100 \mathrm{keV}$ photon energies at PETRA III [2]. Using this same setup we have developed a novel ultrahigh vacuum compatible deposition chamber, that allows for the demanding sample alignment of the thin film sample under vacuum conditions as well as a 180 degree in-plane rotation [4]. Via a rotary feedthrough and bellows combination the surrounding vacuum chamber is not affected by translation of the sample, allowing for film deposition equipment such as a magnetron sputter source. This has been employed at the P07-EH2 beamline with a radio-frequency magnetron sputter source, as pictured in the figure below. We will show how this has been applied to observe the formation of thin films during the initial stages of deposition in real time with subsecond time resolution.

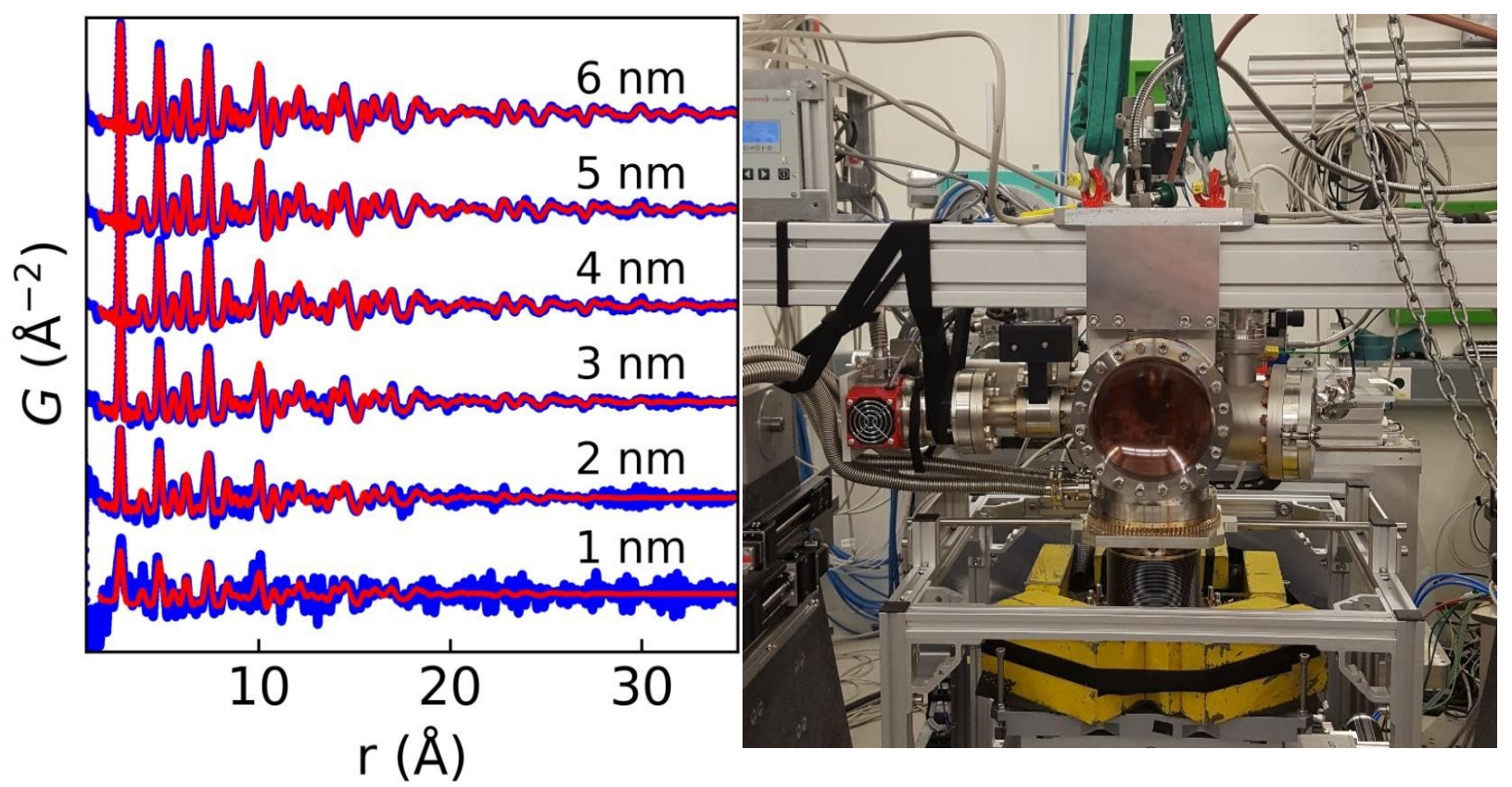

Figure 1. PDFs of a Pt thin film at 10, 20,30, 40,50 and 60 seconds of sputter deposition with approximately $1 \AA /$ s and a picture of the equipment installed on the surface diffractometer at beamline P07-EH2, PETRA III, Hamburg, Germany, as seen from the detector side.

1. K. M. Ø. Jensen et al., IUCrJ 2 (2015), 481

2. A.-C. Dippel et al., IUCrJ 6 (2019), 291

3. K. Stone et al., APL Materials 4 (2016), 076103

4. M. Roelsgaard et al., IUCrJ 6 (2019), 299 